\title{
MicroRNAs tend to synergistically control expression of genes encoding extensively-expressed proteins in humans
}

\author{
Xue Chen ${ }^{1}$, Wei Zhao ${ }^{1}$, Ye Yuan ${ }^{1}$, Yan Bai ${ }^{1}$, Yong Sun ${ }^{1}$, Wenliang Zhu ${ }^{1}$ ， Zhimin Du ${ }^{\text {Corresp. } 1}$ \\ ${ }^{1}$ Department of Pharmacy, the Second Affiliated Hospital of Harbin Medical University (Institute of Clinical Pharmacy, the Heilongjiang Key Laboratory of \\ Drug Research, Harbin Medical University), Harbin, China \\ Corresponding Author: Zhimin Du \\ Email address: dzm1956@126.com
}

Considering complicated microRNA (miRNA) biogenesis and action mechanisms, it was thought so high energy-consuming for a cell to afford simultaneous over-expression of many miRNAs. Thus it prompts that an alternative miRNA regulation pattern on proteinencoding genes must exist, which has characteristics of energy-saving and precise protein output. In this study, expression tendency of proteins encoded by miRNAs' target genes was evaluated in human organ scale, followed by quantitative assessment of miRNA synergism. Expression tendency analysis suggests that universally expressed proteins (UEPs) tend to physically interact in clusters and participate in fundamental biological activities whereas disorderly expressed proteins (DEPS) are inclined to relatively independently execute organ-specific functions. Consistent with this, miRNAs that mainly target UEP-encoding mRNAs, such as miR-21, tend to collaboratively or even synergistically act with other miRNAs in fine-tuning protein output. Synergistic gene regulation may maximize miRNAs' efficiency with less dependence on miRNAs' abundance and overcome the deficiency that targeting plenty of genes by single miRNA makes miRNA-mediated regulation high-throughput but insufficient due to target gene dilution effect. Furthermore, our in vitro experiment verified that merely $25 \mathrm{nM}$ transfection of miR21 be sufficient to influence the overall state of various human cells. Thus miR-21 was identified as a hub in synergistic miRNA-miRNA interaction network. Our findings suggest that synergistic miRNA-miRNA interaction is an important endogenous miRNA regulation mode, which ensures adequate potency of miRNAs at low abundance, especially those implicated in fundamental biological regulation. 

expressed proteins in humans

3 Xue Chen, Wei Zhao, Ye Yuan, Yan Bai, Yong Sun, Wenliang Zhu, Zhimin Du*

4 Department of Pharmacy, the Second Affiliated Hospital of Harbin Medical University (Institute of Clinical

5 Pharmacy, the Heilongjiang Key Laboratory of Drug Research, Harbin Medical University), Harbin, China

6 *To whom correspondence should be addressed. Zhimin Du: Address: 246 Xuefu Road, Harbin 150086,

7 Heilongjiang Province, China; Tel/Fax: 86-451-86605353; E-mail: dzm1956@126.com.

8 


\section{ABSTRACT}

10 Considering complicated microRNA (miRNA) biogenesis and action mechanisms, it was thought so high energy-consuming for a cell to afford simultaneous over-expression of many miRNAs. Thus it prompts that an alternative miRNA regulation pattern on protein-encoding genes must exist, which has characteristics of energy-saving and precise protein output. In this study, expression tendency of proteins encoded by miRNAs' target genes was evaluated in human organ scale, followed by quantitative assessment of miRNA synergism. Expression tendency analysis suggests that universally expressed proteins (UEPs) tend to physically interact in clusters and participate in fundamental biological activities whereas disorderly expressed proteins (DEPs) are inclined to relatively independently execute organ-specific functions. Consistent with this, miRNAs that mainly target UEP-encoding mRNAs, such as miR-21, tend to collaboratively or even synergistically act with other miRNAs in fine-tuning protein output. Synergistic gene regulation may maximize miRNAs' efficiency with less dependence on miRNAs' abundance and overcome the deficiency that targeting plenty of genes by single miRNA makes miRNA-mediated regulation high-throughput but insufficient due to target gene dilution effect. Furthermore, our in vitro experiment verified that merely $25 \mathrm{nM}$ transfection of miR-21 be sufficient to influence the overall state of various human cells. Thus miR-21 was identified as a hub in synergistic miRNA-miRNA interaction network. Our findings suggest that synergistic miRNA-miRNA interaction is an important endogenous miRNA regulation mode, which ensures adequate potency of miRNAs at low abundance, especially those implicated in fundamental biological regulation. 


\section{INTRODUCTION}

MicroRNAs (miRNAs) belong to a super-family of $\sim 22$ nucleotides single-stranded non-coding RNA molecules, which are extensively implicated in pathophysiological activities (Bartel, 2009; Shukla et al., 2011). After a long biogenesis process, functionalities of miRNAs rely on their interaction with argonaute proteins and co-assembling into the RNA-induced silencing complex (RISC). Despite energy-consuming, such a unique gene regulation pattern may be significant to human cells owing to their macro roles in biological regulation (Adlakha \& Seth, 2017; Park et al., 2016; Srinivasan \& Das, 2015).

One miRNA usually physically interacts with hundreds of target genes (Zhou and Yang, 2012), suggesting the advantage of high-throughput and integrative gene regulation (Liu et al., 2016). However, it was also proposed that an inherent deficiency should be not ignored regarding the operation of a 'one to many' system like this. Targeting hundreds of genes undoubtedly dissipates the efficacy of the miRNARISC machine due to the abundance dilution effect of target genes (Arvey et al., 2010). Undoubtedly, comprehensive and effective regulation covering all target genes needs high miRNA output with sufficient RISC machines in which mature miRNA is embedded. Such a strategy may be uneconomical for widespread adoption due to excessive occupancy of cellular energy and material. Actually, the completed human miRNA expression profiles have shown that only a very small portion of miRNAs can afford this way, such as miR-1 that is strongly expressed in heart and miR-122a that is highly expressed in liver (Ritchie et al., 2010). Just recently, it has been revealed that $\sim 10-15 \%$ of human miRNAs are tissue-specific, replying limited cell load capacity for miRNAs (Ludwig et al. 2016).

Indirect and non-physical interactions exist among miRNAs, which forms the basis of the overall effect of miRNA-mediated gene regulation. Several possible patterns of miRNA-miRNA interactions (MMIs) 
50

were uncovered by far. As one miRNA can physically interact with hundreds of target genes, this causes with a great probability that different miRNAs may competitively bind the 3 '-untranslated region of the same gene. Competition for binding is the main and fundamental pattern of miRNA-miRNA interactions (MMIs) in the miRNA world (Jens and Rajewsky, 2015). Expect the competition pattern, different miRNAs may show cooperativity due to being co-regulated by the same transcription factor and targeting genes with functional interconnections ( $\mathrm{Na}$ and Kim, 2013; Shi et al., 2013; Guo et al., 2014). Restricted MMI is another pattern that two miRNAs have completely or partially complementary structures and constitute an endogenous sense and antisense miRNA pair (Guo et al., 2014). Great expression difference of the two miRNAs in a pair was a major feature of this pattern.

Based on our previous knowledge of miRNA regulation (Yuan et al., 2015; Zhu et al., 2011; Zhu et al., 2013), we proposed that miRNAs might adopt an alternative pattern of MMI that promotes a manner of more economical and efficient gene regulation in response to real-time adjustment of cellular signals. It less depends on miRNA abundance and more rely on synergistic miRNA-miRNA collaboration for fine-tuning protein output (Skommer et al., 2014). On the whole, synergistic gene regulation may optimize the regulatory efficacy of miRNAs (Xu et al., 2011). Importantly, it is less expensive in cellular energy consumption compared to extraordinarily high expression of miRNAs. In order to validate this hypothesis, we performed a quantitative assessment of miRNA synergism by calculating miRNA synergy score (Zhu et al., 2013). Benefited from the increasingly clear human protein expression atlas (Uhlén et al., 2015), this method enables us to explore potential principles in miRNA regulation at the dimension of human organs. Additionally, in vitro experiment was performed to show the potential benefit of miRNA synergy. Such efforts are intended to shed novel insights into the biological significance of miRNA-miRNA collaboration 
and provide implications for better understanding the existence of miRNAs in humans.

\section{MATERIALS AND METHODS}

\section{MiRNA-target interactions (MTIs) and protein expression}

Three datasets miRecords (Xiao et al., 2009), miRSel (Naeem et al., 2010) and ExprTargetDB (Gamazon et al., 2010) were used to obtain reliable MTIs in humans. MiRSel is a collection of literature evidence of MTIs. MiRecords and ExprTargetDB belong to secondary tools for MTI prediction, in which integration of different algorithms led to reliable MTI identification. Finally, all MTI data were merged for further analysis. Organ protein expression data were obtained from the human protein atlas (HPA) database (Uhlén et al., 2015). Twelve human organs were included: breast and female reproductive system (BFS), blood and immune system (BIS), central nervous system (CNS), cardiovascular system (CVS), digestive tract (DT), endocrine glands (EG), liver and pancreas (LP), male reproductive system (MS), placenta (P), respiratory system (RS), skin and soft tissues (SS) and urinary tract (UT). A resilient fraction threshold was adopted for expression verification. Specifically, the fraction threshold was set at $70 \%$ for BFS, BIS, CNS, LP and SS; $90 \%$ for DT; $100 \%$ for CVS, EG, MS, P, RS and UT. The information of the level of annotated protein expression was downloaded and imported into Cytoscape v2.8.3 (Smoot et al., 2011). Notably, validated marks 'none', 'low', 'medium' and 'high' representing proteins expression levels were in advance converted into the digitals $0,1,2$ and 3, respectively. Afterwards, coefficient of variation (CV) was used to assess the cellular expression dispersion degree of each miRNA target gene-encoded protein.

\section{Synergy score and skewness}

Potential degree of synergistic collaboration between miRNAs was quantitatively assessed by calculating 
miRNA synergy score as described before (Zhu et al., 2013). For each miRNA, the statistics parameter skewness was used to assess the distribution inclination of the $\log _{2} \mathrm{CV}$ values of protein expression and determine the regulatory tendency of the miRNA (Mardia, 1970). We used $\log _{2} \mathrm{CV}$ instead of CV for skewness calculation, as the distribution of CVs did not pass D'Agostino \& Pearson omnibus normality test (D'Agostino and Pearson, 1973). A smaller skewness value means a tendency of regulating mRNAs encoding uniformly expressed proteins (UEPs); however a higher skewness value suggests that a miRNA tends to fine-tune disorderly expressed proteins (DEPs). File S1 was a four-step protocol to reveal miRNAs that tend to regulate UEP-encoding mRNAs. In this study, UEPs and DEPs refer to proteins with CVs of less than $40 \%$ and CVs of more than $120 \%$, respectively.

\section{Gene ontology (GO) and network topology}

A comparison between UEPs and DEPs was investigated from the GO aspect by applying the online DAVID functional annotation tool (Huang et al., 2009). Briefly, gene official symbols were submitted as gene list and 'Homo Sapiens' was selected as the background. Over-represented GO biological processes, cellular components and molecular functions were considered to be significant only if false discovery rate (FDR) was less than 0.05 . We also investigated network topology characteristics of UEPs and DEPs, respectively. The Cytoscape plugins BisoGenet [18] was used to retrieve, trim, and analyze experimentally validated protein-protein interactions (PPIs).

\section{Cell culture and miRNA transfection}

Human umbilical vein endothelial cell line (HUVEC), breast cancer cell line (MCF7) and hepatic carcinoma cell line (HepG2) were all obtained from the Institute of Biochemistry and Cell Biology, Chinese Academy of Science (Shanghai, China). Cells were maintained in Dulbecco's modified Eagle's medium (DMEM) 
113

114 115 116 117 118 119

supplemented with $10 \%$ fetal bovine serum, $100 \mathrm{U} / \mathrm{mL}$ penicillin and $100 \mu \mathrm{g} / \mathrm{mL}$ streptomycin. After that, cells were incubated at $37{ }^{\circ} \mathrm{C}$ with $5 \% \mathrm{CO}_{2}$ and $95 \%$ air. 24 hours before transfection, cells were transferred to 96-well plates and cultured in fresh medium without antibiotics, According to the manufacturer's instructions, X-treme GENE siRNA transfection reagent (Roche, Switzerland) was used for monotransfection of negative control or miRNA mimic at $25 \mathrm{nM}$. Table S1 listed the sequences of the negative control and miRNA mimics used in this study.

\section{Cell viability assay}

Cell viability was assessed by measuring mitochondrial dehydrogenase activity, using the colorimetric MTT assay, based on the fact that viable cells (but not dead cells) can reduce 3-(4,5-dimethylthiazol-2-yl)2,5-diphenyl tetrazolium bromide (MTT). After miRNA transfection 24 hours, with or without hypoxia treatment, cells were incubated with MTT of $5 \mathrm{mg} / \mathrm{ml}$ at $37{ }^{\circ} \mathrm{C}$ for four hours. Hypoxia was induced by exposing cells to hypoxia condition $\left(1 \% \mathrm{O}_{2}, 94 \% \mathrm{~N}_{2}, 5 \% \mathrm{CO}_{2}\right)$ for 24 hours using a modular incubator. The purple formazan crystal was dissolved with $150 \mu \mathrm{L}$ of dimethyl sulfoxide (DMSO) and added to the cells. The absorbance was measured at $490 \mathrm{~nm}$.

\section{Data statistics}

All data are expressed as mean \pm SEM (Standard Error of Mean). Statistical analysis was performed with Mann-Whitney $U$ test or one-way ANOVA (analysis of variance) followed by Tukey's test for multiple comparisons. Differences were only considered to be significant at $p<0.05$.

\section{RESULTS}

\section{Data collecting and screening}


Data integration identified a total of 11162 target genes and 69618 MTIs for 472 miRNAs from the three databases including miRecords, miRSel, and ExprTargetDB. Only 166 of were retained for further analysis, each of which targeted at least 50 mRNA genes in each of the 12 human organs (Table S2). Totally, they were functionally associated with more than 6328 proteins via 36211 MTIs.

\section{Comparison of UEPs and DEPs in organ expression, network topology and GO terms}

After retrieving the protein expression data from the HPA database, we calculated the CV value for each of the 6328 proteins regarding its HPA-defined abundance ranks in human cells. The majority of the proteins were located with the CV range from $40 \%$ to $120 \%$ (Figure 1A). This distribution characteristic was more clearly observed after $\log 2$ transformation of CVs. Totally, we identified 1340 UEPs and 1115 DEPs. Unlike DEPs, UEPs tend to be more uniformly expressed in human organs, have more interacting neighbors, and constitute closer associations with each other in clusters (Figures 1B-D). Comparably, DEPs are not ubiquitously expressed, and more marginalized in the human PPI network and execute functions in isolation. More than these, GO analysis disclosed that UEPs tend to be intensively involved in gene transcription and related biological processes, be located in the nucleus or nearby, and play roles in the housekeeping functions such as being transcription regulators (Table S3). This result indicates the crucial role of UEPs in the maintenance of basic cell activities. In contrast, DEPs are more implicated in organspecific biological processes and perform molecular functions at the plasma membrane and cytoplasmic organelles.

\section{MiRNAs' regulation tendency on mRNAs encoding UEPs or DEPs is independent of miRNA}

\section{expression}

We investigated the difference of UEPs and DEPs from the miRNA regulation aspect. UEP-encoding 
155

156

157

158

159

160

161

162

163

164

165

166

167

168

169

170

171

172

173

174

175

mRNAs instead of those encoding DEPs are averagely under more dense control of miRNAs in the whole genome context (Figure 2A). Even when MTIs are restricted into one organ such as CVS, preference of miRNAs for UEP-encoding mRNAs is also obvious to observe (Figure 2B). The highest skewness value highlighted miR-133a of its definite trend in regulating DEP-encoding mRNAs in CVS (Figure 2C). Both miR-1 and 133a are cardiac-specific miRNAs. However, we found that they showed different tendencies on gene regulation (Figure 2D). This result suggests that regulating organ-specific genes may be not just a necessary condition for the organ-specific functions of miRNAs, such as miR-1 (Yang et al., 2007).

\section{MiRNAs regulate UEP-encoding mRNAs in a synergistic pattern}

MiRNAs may act synergistically with each other (Xu et al., 2011). With the evolution of the complexity of a biological system, synergism is undoubtedly more advantageous than isolated regulation in terms of management strategy (Corning, 1995; Stelling et al., 2004). Altered expression of only a few numbers of miRNAs caused systemic changes via disrupting synergistic associations between miRNAs (Figures 3A-

C). If all conceivable miRNA pairs are supposed to be synergistic $75.5 \%$ of synergistic miRNA interactions will be affected only when half of all the miRNAs undergo altered expression (Figure 3D). In particular, to those the hubs in the miRNA-miRNA synergistic network, adaptation of this management strategy may be more energy-saving due to plenty of synergistic partners (Figure 3E). Further result indicates that miRNAs with small skewness values comprise most of the synergistic miRNA-miRNA associations in human organs (Figure 4). This finding implies that the molecular behaviors of UEPs, rather than those of DEPs, are under the surveillance of a dense synergistic miRNA regulation network. In this invisible network, several miRNAs should be paid more attention owing to their more powerful and extensive collaboration with other miRNAs (Figure 5). They are miR-1, miR-21, miR-141, miR-155, miR-181b, miR-200a, and miR-206. 
176 Compared with other miRNAs, miR-21 had a significant impact on the whole cell state of the three cell

177 lines at a mono-transfection concentration of $25 \mathrm{nM}$ (Figure 6). MiR-133a tends to regulate DEP-encoding

178 mRNAs implying its relatively isolated action, our in vitro experiment verified its failing to influence the whole cell state of the three cell lines.

\section{DISCUSSION}

The new discovery of miRNAs and other kinds of non-coding RNAs greatly consolidates the dominant position of genes in regulating cellular activities (Cech and Steitz, 2014). Genes are not only carriers and messengers of genetic information, but also direct supervisors on whether such information is accurately translated into phenotypes. Despite lots of efforts it is not still clear about the regulation layer that is constituted by thousands of miRNAs by now (Boettger and Braun, 2012; Stroynowska-Czerwinska et al., 2014). In this study, system-level insight was attempted to shed into the overall effect of miRNAs from the perspective of miRNA-miRNA collaboration.

It was confirmed that abundant expression of target genes produces the abundance dilution effect for miRNA-mediated regulation (Arvey et al., 2010). Denzler and his colleagues further proved that the overall abundance of target genes is generally higher than that of the corresponding miRNA (Denzler et al., 2014). This causes insensitive influence of competitive endogenous RNA (ceRNA)-mediated derepression on miRNA activity (Denzler et al., 2016). The above findings imply that miRNAs may do not perform functions in isolation and collaboration of different miRNAs is a means of effective regulation of gene expression.

Functional studies have confirmed that miRNAs might act synergistically, strongly suggesting 
197

198

199

200

201

202

203

204

205

206

207

208

209

210

211

212

213

214

215

216

217

collaborative gene regulation (Huang et al., 2016; Raut et al., 2016; Xue et al., 2016). The above evidence

is properly consistent with the conclusion inferred by the systems biology analyses performed before (Xu et al., 2011; Zhu et al., 2013). Extensive miRNA-miRNA synergism may exist in the miRNA layer.

Precisely because of this, slight adjustment of miRNAs' abundance can cause an apparent variation in the state of cells. Synergistic miRNAs action allows cells to adjust their live-or-death status more flexibly and rigorously to adapt to internal and external alternation. Due to acting synergistically with other miRNAs, low concentration transfection of miR-21 showed an obvious impact on the viability of all of the studied cell lines. Our result is in agreement with the established knowledge that miR-21 is a well-known miRNA that targets tumor suppressor genes and was shown experimentally in many studies to control apoptosis (Buscaglia and Li, 2011). This finding also implies that altered miRNA expression may not only independently affect the translation of its own target gene products but also produces a radiative impact on other miRNAs that have synergistic associations with it. Put briefly, miRNA synergy amplifies the effects of miRNA expression alteration. As cell viability assay is only one way of measuring effects of miRNAs on the cell state and it cannot be excluded that the other examined miRNAs with high potential to regulate UEP-encoding mRNAs can affect the cell state in another way. However, further studies are definitely needed to investigate the miRNAs through other experimental methods.

Functional execution of miRNAs requires high cellular energy consumption as a prerequisite (Shukla et al., 2011). Besides of miRNA regulatory crosstalk (Jens and Rajewsky, 2015), miRNA synergy may represent an alternative pattern of energetically optimal miRNA-mediated regulation of post-transcriptional gene expression. However, we supposed that this obvious advantage brought by miRNA synergy might rely heavily on the complexity of the biological system. As miRNA synergy score calculation disclosed, 
218

219

220

221

222

223

224

225

226

227

228

229

230

231

232

233

234

235

236

237

238

only dense PPIs between target gene products could lead to meaningful miRNA synergy (Zhu et al., 2011).

This explains well that one can expect strong synergistic potential of the cardic-specific miRNA miR-1 instead of miR-133a. Our findings indicate that miR-1 has a tendency to regulate UEP-encoding mRNAs whereas miR-133a tends to regulate DEP-encoding mRNAs. A deeper insight is that only complex life forms such as human can afford the coexistence of hundreds of miRNAs in a cell owing to the intricate PPI network that is an essential condition for miRNA synergy to occur.

Besides, our results further point out that miRNA synergism may be associated with the organ expression of target gene products. UEPs that have broad and balanced expression in human organs are subject to oversupervision by synergistic miRNA regulation. Broad and balanced expression of UEPs is a reasonable reflection of their important biological roles in cellular activities such as significant participation in gene transcription. Uniformed expression of large number of proteins throughout human organs implies that different cells share a similar basic state, which ensures that the communication between cells is relatively equal in the physiological environment. Strengthened synergistic miRNA regulation on UEP-encoding mRNAs suggests that miRNA synergism should contribute to the maintaining of their uniformed expression throughout human organs. Due to allowing cells more finely tune the protein abundance of UEPs, miRNA synergism leads to more economical adaptation of cells to the intracellular and extracellular environments.

The complexity of the biological system is described by the following characteristics such as robustness, redundancy, and crosstalk (Jia et al., 2009). Biological robustness lies in that altered expression of a single gene may be insufficient to effectively affect the overall system (Kitano, 2004). Although this guarantees the relative stability of a biological system, it makes cells difficult to adapt to the external environment changes in a timely manner. Due to the existence of functional linkages between system components, cells 
239

240

241

242

243

244

245

246

247

248

249

250

251

252

253

254

255

256

257

258

259

cannot merely change one component but keep others unchanged when faced with altered conditions. For instance, as cells encounter excessive oxidative stress, adjustment of the overall cellular signals could be observed (Chandra et al., 2000; Kiffin et al., 2006; Reuter et al., 2010). Robustness and adaptability are mutually contradictory. Biological system requires an integrated gene/protein management strategy for coordinating diverse biological signals. By targeting hundreds of mRNA messengers, single miRNA is in a position to accomplish integrated gene regulation (Backes et al., 2017). Compared to mRNA-mediated protein expression control, indirect regulation by miRNAs is high-throughput (Baek et al., 2008). This may be the biological significance of miRNAs' presence in human biology. More importantly, the unique synergistic action mechanism of miRNAs provides richer control skills for cells and better overcomes the huge energy consumption in biosynthesis of miRNAs and assembly of RISC protein complex.

Undoubtedly, more in-depth exploration of constituent factors for effective miRNA target site (Agarwal et al., 2015) would greatly promote our understanding of synergistic interactions between miRNAs. Differential 3'-untranslated region isoforms may lead to inconsistent target gene profiles of a miRNA in different types of cells (Nam et al., 2014). Combined with the fact that $\sim 10-15 \%$ of human miRNAs are tissue-specific (Ludwig et al., 2016), this finding implies that synergistic collaboration between miRNAs may be cell-type-specific or tissue-specific. However, more experimental researches are definitely to explore this.

In conclusion, no longer depending on self-expression lets miRNA synergism maximize the effectiveness of fine-tuning protein output. For complex biological systems, miRNA synergism seems to be a very energy-economical solution by which cells can better deal with the contradictory relationship between system robustness and system adaptability. Our findings provide a new understanding of the biological 
260 261

262

263

264

265

266

267

268

269

270

271

272

273

274

275

276

277

278

279

280

significance of miRNAs in the organ scale.

\section{REFERENCES}

Adlakha YK, Seth P. 2017. The expanding horizon of MicroRNAs in cellular reprogramming. Progress in Neurobiology 148:21-39 DOI 10.1016/j.pneurobio.2016.11.003.

Agarwal V, Bell GW, Nam JW, Bartel DP. 2015. Predicting effective microRNA target sites in mammalian mRNAs. Elife 4 DOI 10.7554/eLife.05005.

Arvey A, Larsson E, Sander C, Leslie CS, Marks DS. 2010. Target mRNA abundance dilutes microRNA and siRNA activity. Molecular Systems Biology 6:363. DOI 10.1038/msb.2010.24.

Backes C, Kehl T, Stöckel D, Fehlmann T, Schneider L, Meese E, Lenhof HP, Keller A. 2017. miRPathDB: a new dictionary on microRNAs and target pathways. Nucleic Acids Research 45(D1):D90D96 DOI 10.1093/nar/gkw926.

Baek D, Villén J, Shin C, Camargo FD, Gygi SP, Bartel DP. 2008. The impact of microRNAs on protein output. Nature 455(7209):64-71 DOI 10.1038/nature07242.

Bartel DP. 2009. MicroRNAs: target recognition and regulatory functions. Cell 136(2):215-233 DOI 10.1016/j.cell.2009.01.002.

Boettger T, Braun T. 2012. A new level of complexity: the role of microRNAs in cardiovascular development. Circulation Research 110(7):1000-1013 DOI 10.1161/CIRCRESAHA.111.247742.

Buscaglia LE, Li Y. 2011. Apoptosis and the target genes of microRNA-21. Chinese Journal of Cancer 30(6):371-380 DOI 10.5732/cjc.011.10132.

Chandra J, Samali A, Orrenius S. 2000. Triggering and modulation of apoptosis by oxidative stress. Free 
281

282

283

284

285

286

287

288

289

290

291

292

293

294

295

296

297

298

299

300

301

Radical Biology and Medicine 29(3-4):323-333 DOI 10.1016/S0891-5849(00)00302-6.

Corning PA. 1995. Synergy and self-organization in the evolution of complex systems. Systems Research 12:89-121.

Cech TR, Steitz JA. 2014. The noncoding RNA revolution-trashing old rules to forge new ones. Cell 157(1):77-94 DOI 10.1016/j.cell.2014.03.008.

D'Agostino R, Pearson ES. 1973. Tests for Departure from Normality. Empirical Results for the Distributions of b2 and $\sqrt{ } \mathrm{b} 1$. Biometrika 60:613-622.

Denzler R, Agarwal V, Stefano J, Bartel DP, Stoffel M. 2014. Assessing the ceRNA hypothesis with quantitative measurements of miRNA and target abundance. Molecular Cell 54(5):766-776 DOI 10.1016/j.molcel.2014.03.045.

Denzler R, McGeary SE, Title AC, Agarwal V, Bartel DP, Stoffel M. 2016. Impact of MicroRNA Levels, Target-Site Complementarity, and Cooperativity on Competing Endogenous RNA-Regulated Gene Expression. Molecular Cell 64(3):565-579 DOI 10.1016/j.molcel.2016.09.027.

Gamazon ER, Im HK, Duan S, Lussier YA, Cox NJ, Dolan ME, Zhang W. 2010. Exprtarget: an integrative approach to predicting human microRNA targets. PLoS One 5(10):e13534 DOI 10.1371/journal.pone.0013534.

Guo L, Zhao Y, Yang S, Zhang H, Chen F. 2014. Integrative analysis of miRNA-mRNA and miRNAmiRNA interactions. BioMed Research International 2014:907420 DOI 10.1155/2014/907420.

Huang da W, Sherman BT, Lempicki RA. 2009. Systematic and integrative analysis of large gene lists using DAVID bioinformatics resources. Nature Protocols 4(1):44-57 DOI 10.1038/nprot.2008.211.

Huang W, Tian SS, Hang PZ, Sun C, Guo J, Du ZM. 2016. Combination of microRNA-21 and 
302 303

304

305

306

307

308

309

310

311

312

313

314

315

316

317

318

319

320

321

322

microRNA-146a Attenuates Cardiac Dysfunction and Apoptosis During Acute Myocardial Infarction in Mice. Molecular Therapy-Nucleic Acids 5:e296 DOI 10.1038/mtna.2016.12.

Jens M, Rajewsky N. 2015. Competition between target sites of regulators shapes post-transcriptional gene regulation. Nature Reviews Genetics 16(2):113-126 DOI 10.1038/nrg3853.

Jia J, Zhu F, Ma X, Cao Z, Cao ZW, Li Y, Li YX, Chen YZ. 2009. Mechanisms of drug combinations: interaction and network perspectives. Nature Reviews Drug Discovery 8(2):111-128 DOI 10.1038/nrd2683.

Kiffin R, Bandyopadhyay U, Cuervo AM. 2006. Oxidative stress and autophagy. Antioxidants \& Redox Signaling 8(1-2):152-162 DOI 10.1089/ars.2006.8.152.

Kitano H. 2004. Biological robustness. Nature Reviews Genetics 5(11):826-837 DOI 10.1038/nrg1471.

Liu Z, Guo Y, Pu X, Li M. 2016. Dissecting the regulation rules of cancer-related miRNAs based on network analysis. Scientific Reports 6:34172 DOI 10.1038/srep34172.

Ludwig N, Leidinger P, Becker K, Backes C, Fehlmann T, Pallasch C, Rheinheimer S, Meder B, Stähler C, Meese E, Keller A. 2016. Distribution of miRNA expression across human tissues. Nucleic Acids Research 44(8):3865-3877 DOI 10.1093/nar/gkw116.

Mardia KV. 1970. Measures of multivariate skewness and kurtosis with applications. Biometrika 57:519530.

Martin A, Ochagavia ME, Rabasa LC, Miranda J, Fernandez-de-Cossio J, Bringas R. 2010. BisoGenet: a new tool for gene network building, visualization and analysis. BMC Bioinformatics 11:91 DOI 10.1186/1471-2105-11-91.

Na YJ, Kim JH. 2013. Understanding cooperativity of microRNAs via microRNA association networks. BMC Genomics 14 Suppl 5:S17 DOI 10.1186/1471-2164-14-S5-S17. 
323

324

325

326

327

328

329

330

331

332

333

334

335

336

337

338

339

340

341

342

343

Naeem H, Küffner R, Csaba G, Zimmer R. 2010. miRSel: automated extraction of associations between microRNAs and genes from the biomedical literature. BMC Bioinformatics 11:135 DOI 10.1186/14712105-11-135.

Nam JW, Rissland OS, Koppstein D, Abreu-Goodger C, Jan CH, Agarwal V, Yildirim MA, Rodriguez A, Bartel DP. 2014. Global analyses of the effect of different cellular contexts on microRNA targeting. Molecular Cell 53(6):1031-1043 DOI 10.1016/j.molcel.2014.02.013.

Park JH, Theodoratou E, Calin GA, Shin JI. 2016. From cell biology to immunology: Controlling metastatic progression of cancer via microRNA regulatory networks. Oncoimmunology 5(11):e1230579

DOI 10.1080/2162402X.2016.1230579.

Raut SK, Singh GB, Rastogi B, Saikia UN, Mittal A, Dogra N, Singh S, Prasad R, Khullar M. 2016. miR-30c and miR-181a synergistically modulate p53-p21 pathway in diabetes induced cardiac hypertrophy. Molecular and Cellular Biochemistry 417(1-2):191-203 DOI 10.1007/s11010-016-2729-7.

Reuter S, Gupta SC, Chaturvedi MM, Aggarwal BB. 2010. Oxidative stress, inflammation, and cancer: how are they linked? Free Radical Biology and Medicine 49(11):1603-1616 DOI 10.1016/j.freeradbiomed.2010.09.006.

Ritchie W, Flamant S, Rasko JE. 2010. mimiRNA: a microRNA expression profiler and classification resource designed to identify functional correlations between microRNAs and their targets. Bioinformatics 26(2):223-227 DOI 10.1093/bioinformatics/btp649.

Shukla GC, Singh J, Barik S. 2011. MicroRNAs: Processing, Maturation, Target Recognition and Regulatory Functions. Molecular and Cellular Pharmacology 3(3):83-92 DOI 10.4255/mcpharmacol.11.13. 
344

345

346

347

348

349

350

351

352

353

354

355

356

357

358

359

360

361

362

363

364

Skommer J, Rana I, Marques FZ, Zhu W, Du Z, Charchar FJ. 2014. Small molecules, big effects: the role of microRNAs in regulation of cardiomyocyte death. Cell Death and Disease 5:e1325 DOI 10.1038/cddis.2014.287.

Shi H, Xu J, Zhang G, Xu L, Li C, Wang L, Zhao Z, Jiang W, Guo Z, Li X. 2013. Walking the interactome to identify human miRNA-disease associations through the functional link between miRNA targets and disease genes. BMC System Biology 7:101 DOI 10.1186/1752-0509-7-101.

Shukla GC, Singh J, Barik S. 2011. MicroRNAs: Processing, Maturation, Target Recognition and Regulatory Functions. Molecular and Cellular Pharmacology 3(3):83-92.

Smoot ME, Ono K, Ruscheinski J, Wang PL, Ideker T. 2011. Cytoscape 2.8: new features for data integration and network visualization. Bioinformatics 27(3):431-432 DOI 10.1093/bioinformatics/btq675.

Srinivasan H, Das S. 2015. Mitochondrial miRNA (MitomiR): a new player in cardiovascular health. Canadian Journal of Physiology and Pharmacology 93(10):855-861 DOI 10.1139/cjpp-2014-0500.

Stelling J, Sauer U, Szallasi Z, Doyle FJ 3rd, Doyle J. 2004. Robustness of cellular functions. Cell 118(6):675-685 DOI 10.1016/j.cell.2004.09.008.

Stroynowska-Czerwinska A, Fiszer A, Krzyzosiak WJ. 2014. The panorama of miRNA-mediated mechanisms in mammalian cells. Cellular and Molecular Life Sciences 71(12):2253-2270 DOI 10.1007/s00018-013-1551-6.

Uhlén M, Fagerberg L, Hallström BM, Lindskog C, Oksvold P, Mardinoglu A, Sivertsson Å, Kampf C, Sjöstedt E, Asplund A, Olsson I, Edlund K, Lundberg E, Navani S, Szigyarto CA, Odeberg J, Djureinovic D, Takanen JO, Hober S, Alm T, Edqvist PH, Berling H, Tegel H, Mulder J, Rockberg J, Nilsson P, Schwenk JM, Hamsten M, von Feilitzen K, Forsberg M, Persson L, Johansson F, 
365

366

367

368

369

370

371

372

373

374

375

376

377

378

379

380

381

382

383

384

385

Zwahlen M, von Heijne G, Nielsen J, Pontén F. Proteomics. 2015. Tissue-based map of the human

proteome. Science 347(6220):1260419 DOI 10.1126/science.1260419.

Xu J, Li CX, Li YS, Lv JY, Ma Y, Shao TT, Xu LD, Wang YY, Du L, Zhang YP, Jiang W, Li CQ,

Xiao Y, Li X. 2011. MiRNA-miRNA synergistic network: construction via co-regulating functional modules and disease miRNA topological features. Nucleic Acids Research 39(3):825-836 DOI $10.1093 / \mathrm{nar} / \mathrm{gkq} 832$.

Xiao F, Zuo Z, Cai G, Kang S, Gao X, Li T. 2009 miRecords: an integrated resource for microRNAtarget interactions. Nucleic Acids Research 37(Database issue):D105-D110 DOI 10.1093/nar/gkn851.

Xue Q, Yu C, Wang Y, Liu L, Zhang K, Fang C, Liu F, Bian G, Song B, Yang A, Ju G, Wang J. 2016. miR-9 and miR-124 synergistically affect regulation of dendritic branching via the AKT/GSK3 $\beta$ pathway by targeting Rap2a. Scientific Reports 6:26781 DOI 10.1038/srep26781.

Yang B, Lin H, Xiao J, Lu Y, Luo X, Li B, Zhang Y, Xu C, Bai Y, Wang H, Chen G, Wang Z. 2007. The muscle-specific microRNA miR-1 regulates cardiac arrhythmogenic potential by targeting GJA1 and KCNJ2. Nature Medicine 13(4):486-491 DOI 10.1038/nm1569.

Yuan D, Cui X, Wang Y, Zhao Y, Li H, Hu S, Chu X, Li Y, Li Q, Liu Q, Zhu W. 2015. Enrichment Analysis Identifies Functional MicroRNA-Disease Associations in Humans. PLoS One 10(8):e0136285

DOI 10.1371/journal.pone.0136285.

Zhou X, Yang PC. 2012. MicroRNA: a small molecule with a big biological impact. Microrna 1(1):1 DOI $10.2174 / 2211536611201010001$.

Zhu W, Yang L, Du Z. 2011. MicroRNA regulation and tissue-specific protein interaction network. PLoS One 6(9):e25394 DOI 10.1371/journal.pone.0025394. 
386

387

388

389

390

391

392

393

394

395

396

397

398

399

400

401

402

403

404

405

406

Zhu W, Yang L, Shan H, Zhang Y, Zhou R, Su Z, Du Z. 2011. MicroRNA expression analysis: clinical advantage of propranolol reveals key microRNAs in myocardial infarction. PLoS One 6(2):e14736. DOI 10.1371/journal.pone.0014736.

Zhu W, Zhao Y, Xu Y, Sun Y, Wang Z, Yuan W, Du Z. 2013. Dissection of protein interactomics highlights microRNA synergy. PLoS One 8(5):e63342 DOI 10.1371/journal.pone.0063342.

\section{FIGURE LEGENDS}

Figure 1. Overview of organ expression of proteins encoded by miRNA targets. A. Frequency distribution of coefficient of variation $(\mathrm{CV})$ of protein expression abundance in 12 human organs. The right upper showed the frequency distribution of $\log _{2} \mathrm{CV}$. The CV interval from $40 \%$ to $120 \%$ was highlighted with a gray box. B. The box and whiskers plot of organ expression percentage of UEPs and DEPs. C. Comparison of the average number of neighbors of UEPs and DEPs. $* * * p<0.001$, UEPs versus DEPs; D. Comparison of cluster coefficient of UEPs and DEPs. $* * * p<0.001$, UEPs versus DEPs. UEPs: uniformly expressed proteins; DEPs: disorderly expressed proteins.

Figure 2. Regulation density and tendency of miRNAs on UEP-encoding mRNAs and DEP-encoding mRNAs. A. Comparison of miRNA density on UEP-encoding mRNAs and DEP-encoding mRNAs. The calculated miRNA density refers to miRNA target sites within only 3' untranslated regions of mRNAs. B. Distribution of the number of miRNAs per target genes that encode proteins expressed in the cardiovascular system. ${ }^{* *} p<0.01, * * * p<0.001$, UEP-encoding mRNAs versus DEP-encoding mRNAs. C. Skewness assessment of miRNAs in the cardiovascular system (CVS). D. Distribution of expression $\log _{2} \mathrm{CVs}$ of proteins encoded by miR-133a and miR-1 target genes. The red dotted line at $\log _{2} \mathrm{CV}$ of -1.32 corresponds 
407

408

409

410

411

412

413

414

415

416

417

418

419

420

421

422

423

424

425

426

427

to the CV value of $40 \%$. CV: coefficient of variation. UEPs: uniformly expressed proteins; DEPs: disorderly

expressed proteins.

Figure 3. Influence of altered expression and synergy on the overall miRNA regulation. A-C.

Percentage of the affected miRNAs and synergistic miRNA interactions at different number of differently

expressed miRNAs. $n=1$ means that one miRNA is dysregulated; $n=3$ or 5 means that three or five miRNAs

are simultaneously dysregulated. D. Percentage of the affected miRNAs and synergistic miRNA

interactions by altering miRNA expression. E. Synergistic miRNA-miRNA interactions in cardiovascular

system. The red, blue and gray lines represent interactions with synergy scores of $>2.0,>1.5$ and $>1.0$,

respectively. Two hubs in the synergistic miRNA interaction network miR-1 and miR-21 were highlighted

as big nodes.

Figure 4. Constitution share of low and high skewness miRNAs for top 500 (A), top 100 (B) and top

50 (C) synergistic miRNA interactions in 12 human organs. *** $p<0.001$, HH versus LL; LL, LH and

HH represent synergies between low skewness miRNAs, between low skewness miRNAs and high

skewness miRNAs, and between high skewness miRNAs, respectively.

Figure 5. Heatmap of mean synergy scores of miRNAs with skewness values of $<0.3$ in 12 human

organs. BFS: Breast and female reproductive system (female tissue); BIS: Blood and immune system

(hematopoietic); CNS: Central nervous system (brain); CVS: Cardiovascular system (heart and blood vessels); DT: Digestive tract (GI-tract); EG: Endocrine glands; LP: Liver and pancreas; MS: Male reproductive system (male tissues); P: Placenta; RS: Respiratory system (lung); SS: Skin and soft tissues; UT: Urinary tract (kidney and bladder).

Figure 6. Cell viability assay of miRNA transfection on cell lines of HUVEC (A), MCF7 (B), and 
428 HepG2 (C). ${ }^{* *} p<0.01$, versus NC+Hypoxia (HUVEC); ${ }^{*} p<0.05$, versus NC (MCF7 and HepG2); NC:

429 negative control; $\mathrm{n}=6$.

430

\section{SUPPLEMENTAL INFORMATION}

Table S1. Sequences of the negative control and miRNA mimics used in this study.

Table S2. Distribution of miRNA target genes in the 12 human organs and within the whole genome.

Table S3. Result of gene ontology (GO) analysis by using DAVID.

File S1. A four-step protocol to reveal miRNAs that tend to regulate UEP-encoding mRNAs. 


\section{Figure 1}

Figure 1. Overview of organ expression of proteins encoded by miRNA target.

A. Frequency distribution of coefficient of variation (CV) of protein expression abundance in 12 human organs. The right upper showed the frequency distribution of $\log _{2} C V$. The CV interval from $40 \%$ to $120 \%$ was highlighted with gray box. B. The box and whiskers plot of organ expression percentage of UEPs and DEPs. C. Comparison of average number of neighbors of UEPs and DEPs. $* * * p<0.001$, UEPs versus DEPs; D. Comparison of cluster coefficient of UEPs and DEPs. $* * * p<0.001$, UEPs versus DEPs. UEPs: uniformly expressed proteins; DEPs: disorderly expressed proteins. 


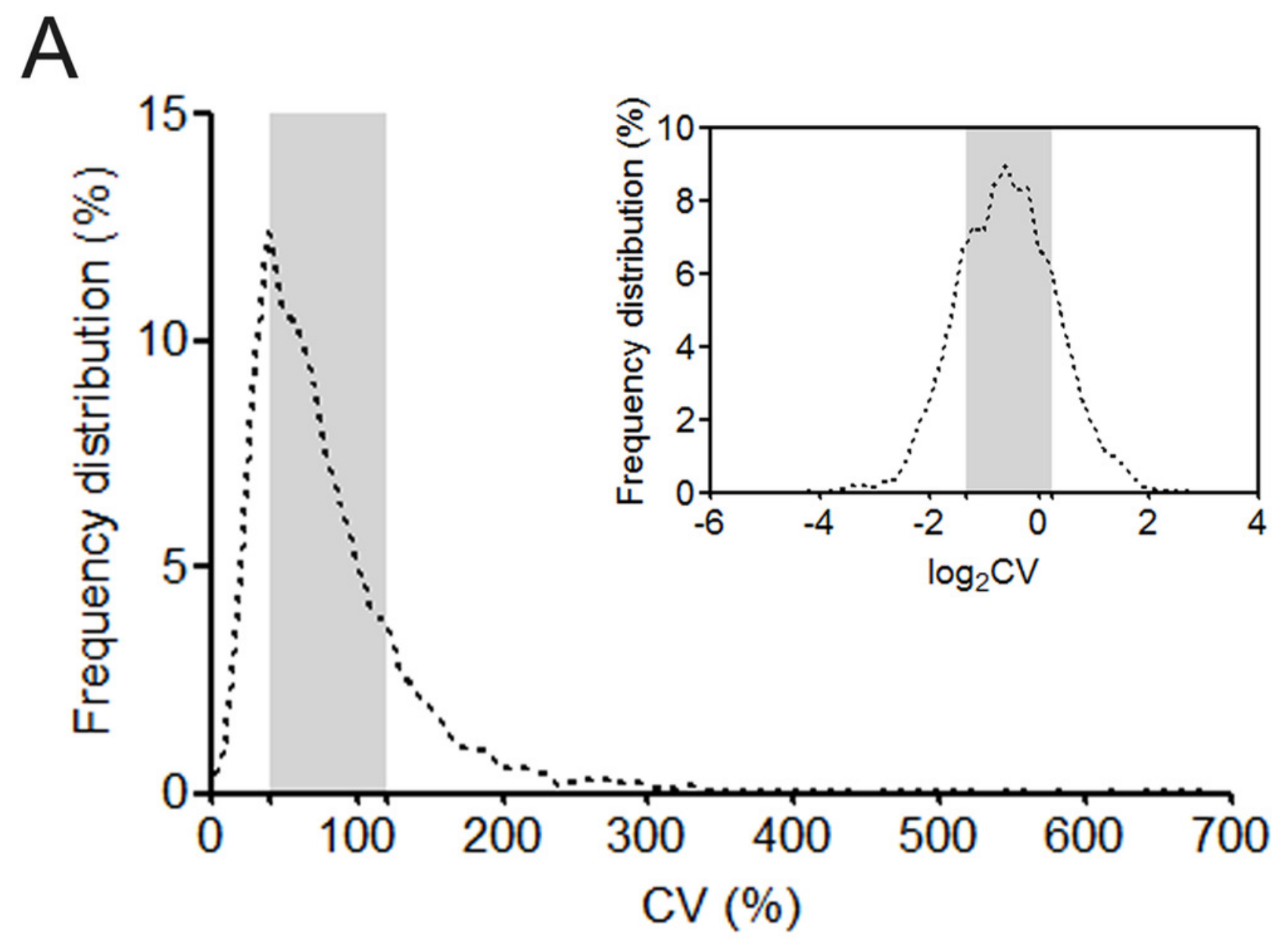

B
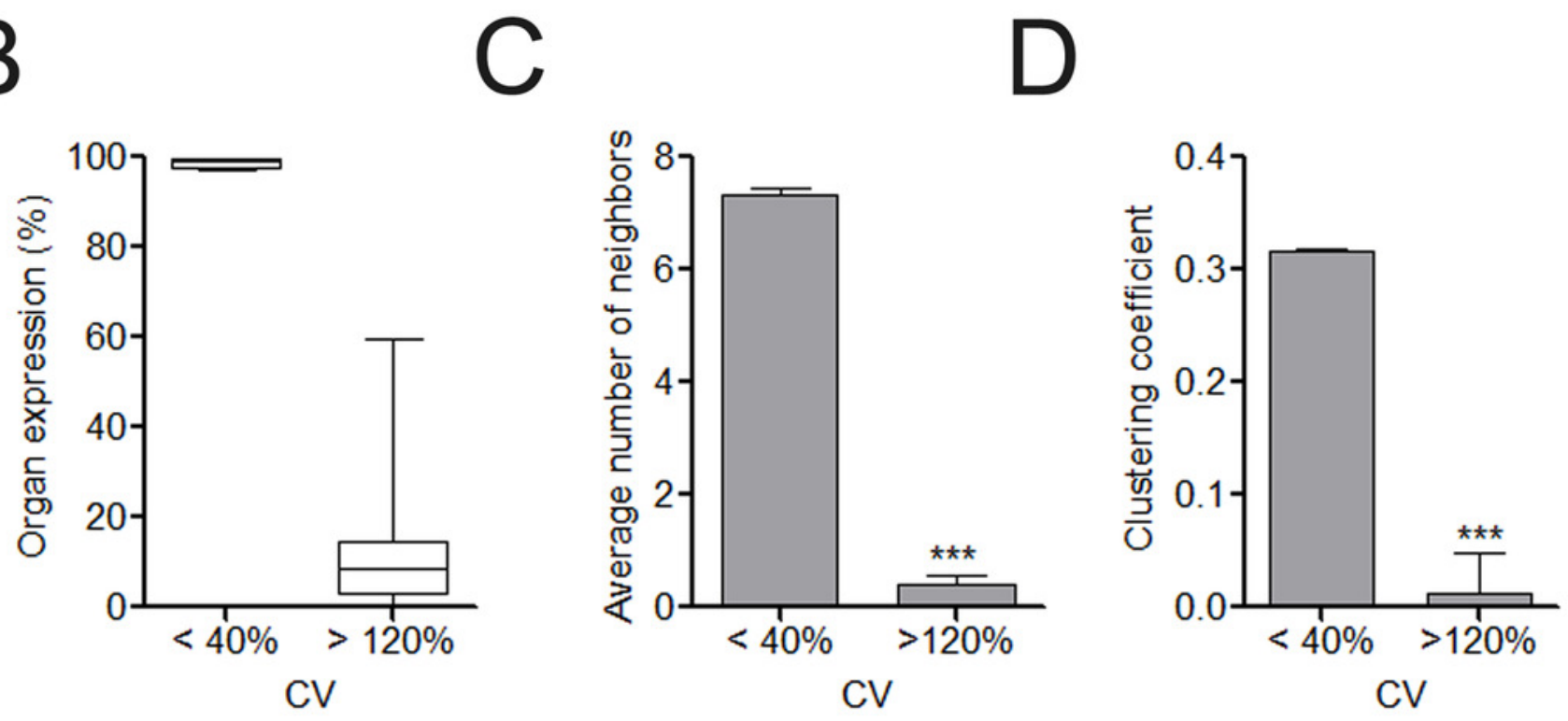


\section{Figure 2}

Figure 2. Regulation density and tendency of miRNAs on UEP-encoding genes and DEPencoding genes.

A. Comparison of miRNA density on UEP-encoding genes and DEP-encoding genes. The calculated miRNA density refers to miRNA target sites within only 3' untranslated regions of genes. B. Distribution of the number of miRNAs per target genes that encode proteins expressed in cardiovascular system. $* * p<0.01, * * * p<0.001$, UEP-encoding genes versus DEP-encoding genes. C. Skewness assessment of miRNAs in cardiovascular system (CVS). D. Distribution of expression $\log _{2} \mathrm{CV}$ s of proteins encoded by miR-133a and miR-1 target genes. The red dotted line at $\log _{2} C V$ of -1.32 corresponds to the $\mathrm{CV}$ value of $40 \%$. CV: coefficient of variation. UEPs: uniformly expressed proteins; DEPs: disorderly expressed proteins. 


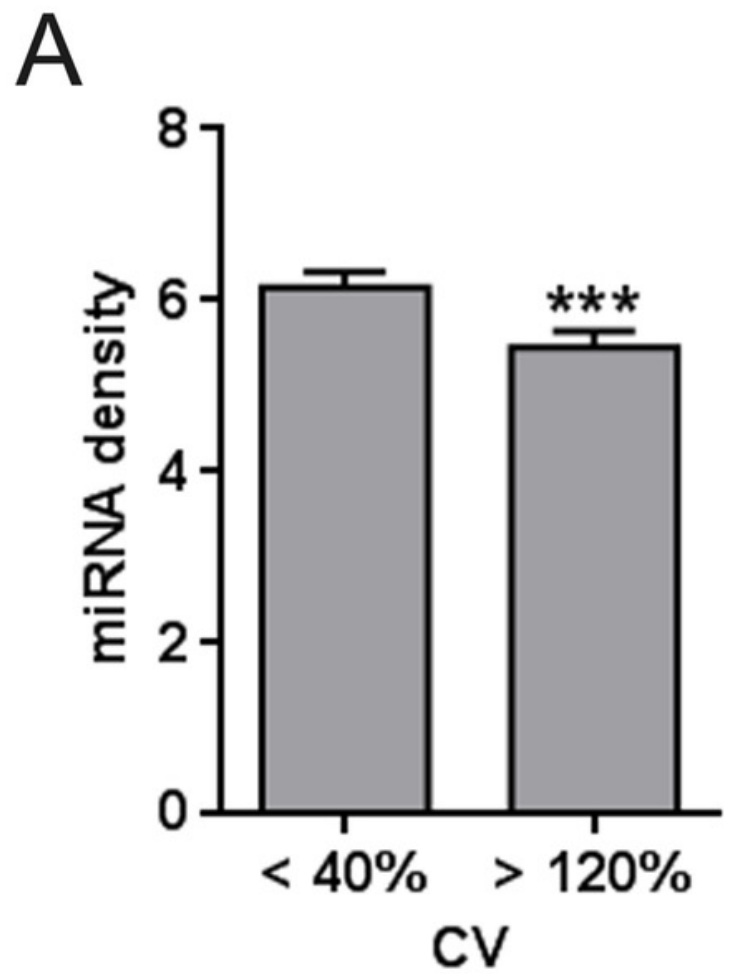

B
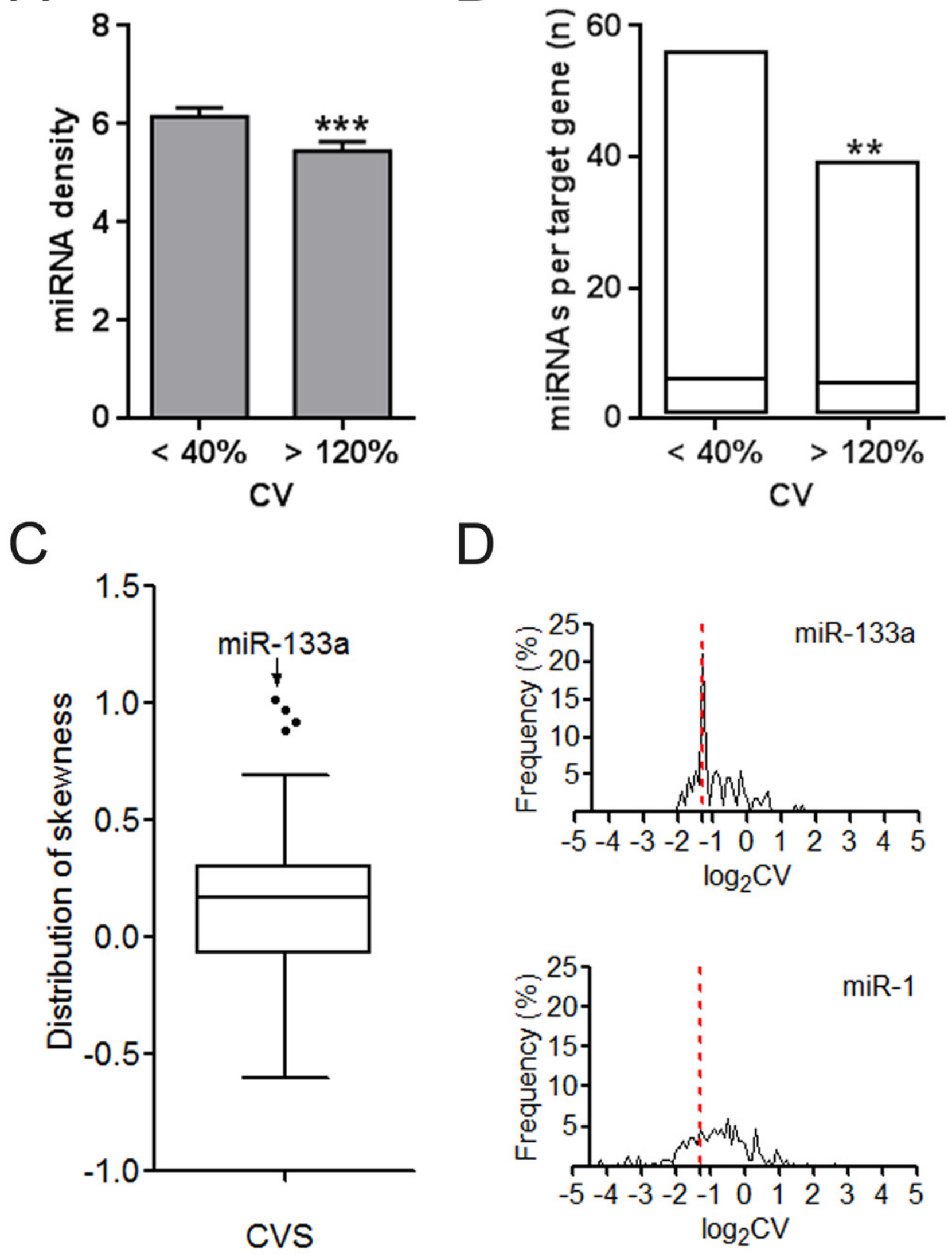


\section{Figure 3}

Figure 3. Influence of altered expression and synergy on the overall miRNA regulation.

A-C. Percentage of the affected miRNAs and synergistic miRNA interactions at different number of differently expressed miRNAs. $n=1,3$, or 5 means that one, three, or five miRNAs are simultaneously dysregulated. D. Percentage of the affected miRNAs and synergistic miRNA interactions by altered miRNA expression. E. Synergistic miRNA-miRNA interactions in cardiovascular system. The red, blue and gray lines represent interactions with synergy scores of $>2.0,>1.5$ and $>1.0$, respectively. Two hubs in the synergistic miRNA interaction network miR-1 and miR-21 were highlighted as big nodes. 
A

C
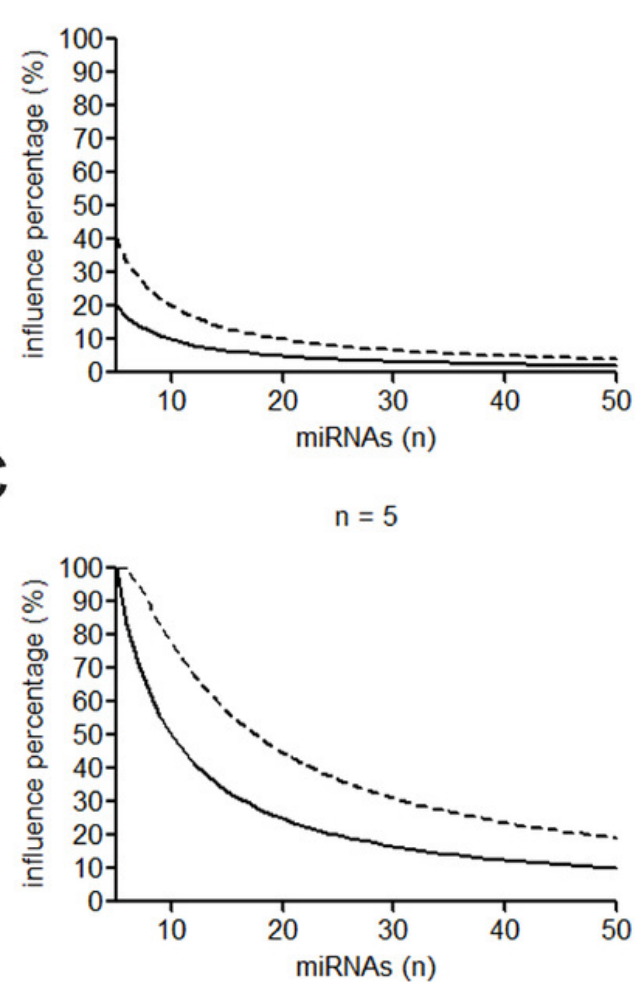

B

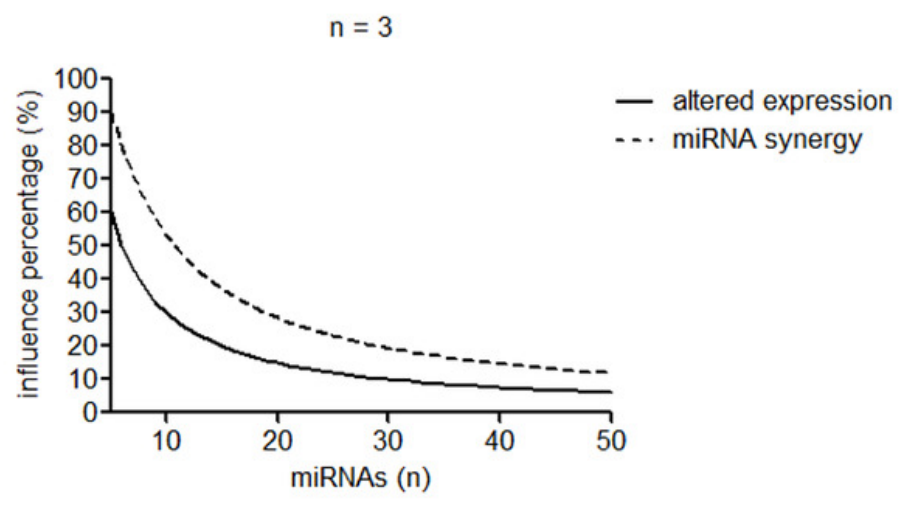

D

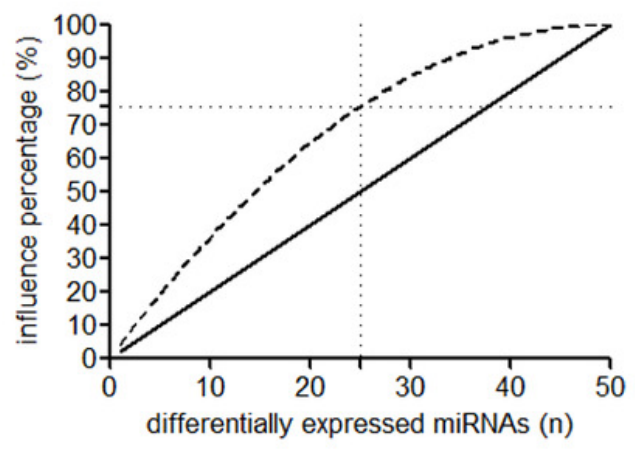

E

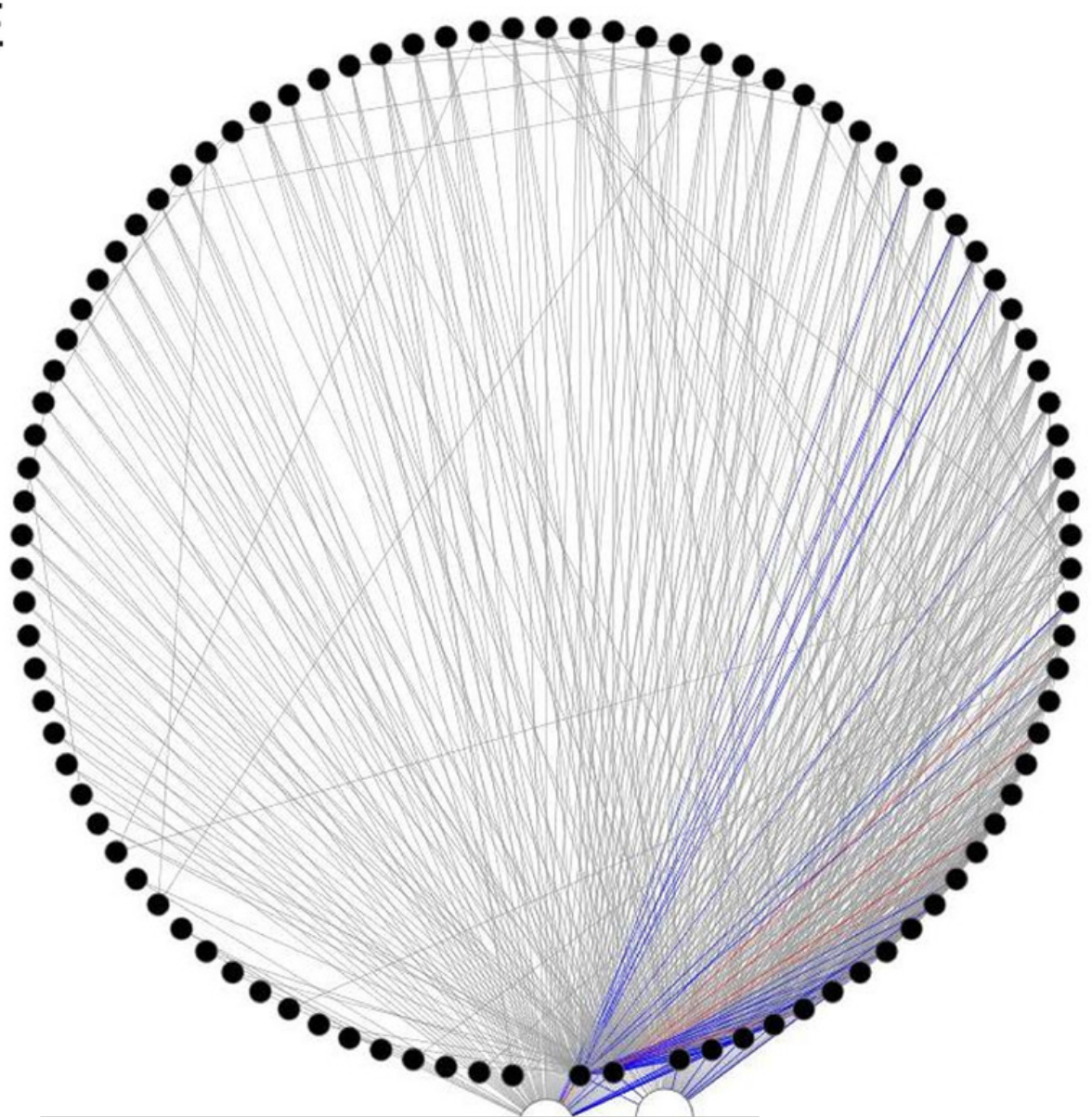

Peer) reviewing PDF | (2017:03:16576:2:0:NEW 13Ju/2017)

miR-21 miR-1 


\section{Figure 4}

Figure 4. Constitution share of low and high skewness miRNAs for top 500 (A), top 100 (B) and top 50 (C) synergistic miRNA interactions in 12 human organs.

${ }^{* * *} p<0.001, \mathrm{HH}$ versus LL; LL, LH and HH represent synergies between low skewness miRNAs, between low skewness miRNAs and high skewness miRNAs, and between high skewness miRNAs, respectively. 
A

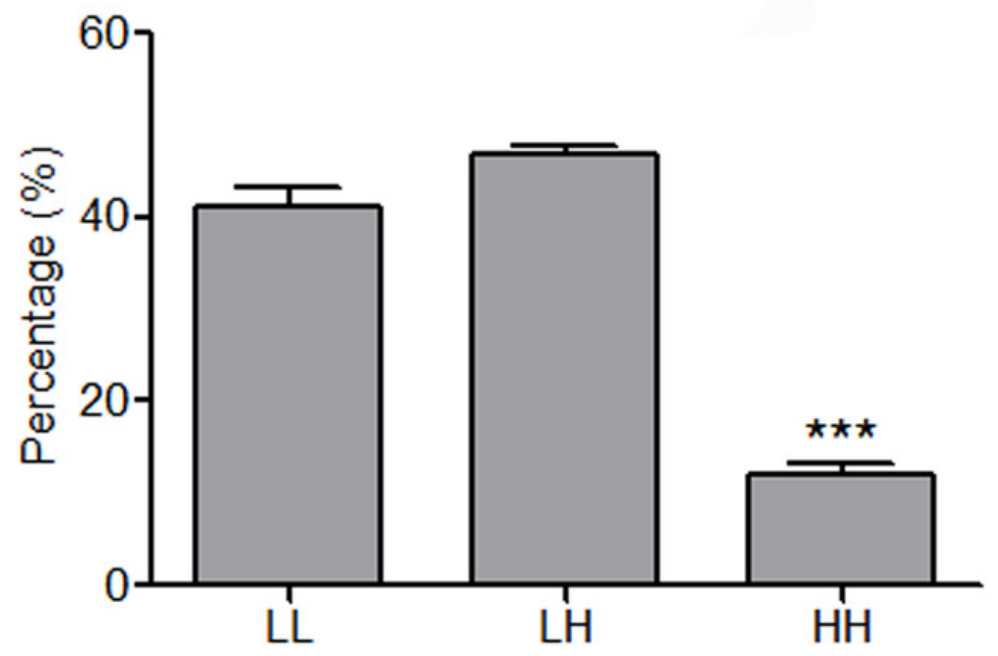

B

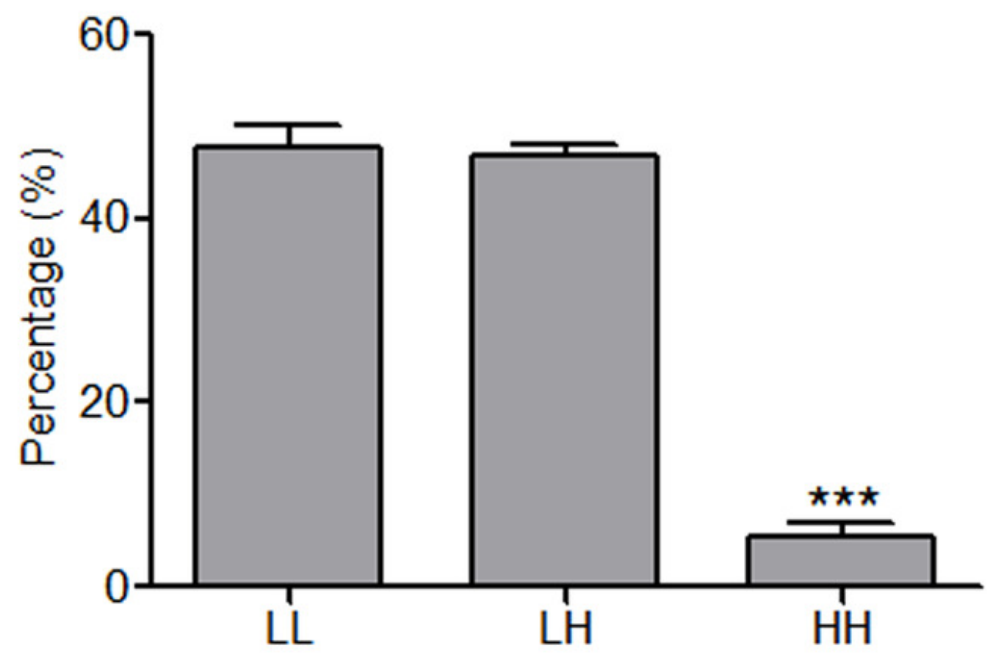

C

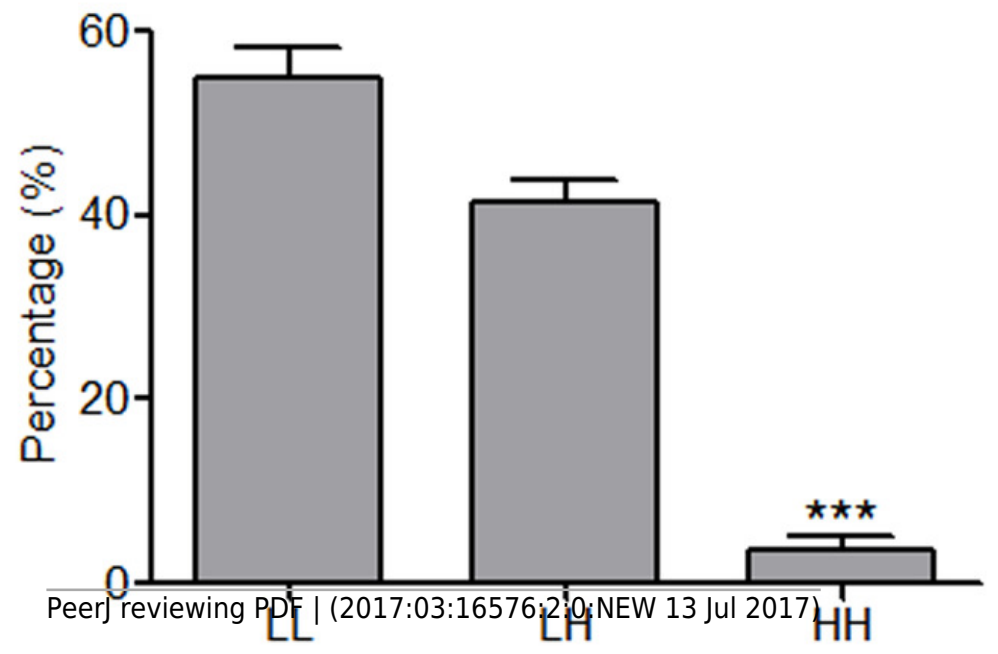




\section{Figure 5}

Figure 5. Heatmap of mean synergy scores of miRNAs with skewness values of $<0.3$ in 12 human organs.

BFS: Breast and female reproductive system (female tissue); BIS: Blood and immune system (hematopoietic); CNS: Central nervous system (brain); CVS: Cardiovascular system (heart and blood vessels); DT: Digestive tract (GI-tract); EG: Endocrine glands; LP: Liver and pancreas; MS: Male reproductive system (male tissues); P: Placenta; RS: Respiratory system (lung); SS: Skin and soft tissues; UT: Urinary tract (kidney and bladder).

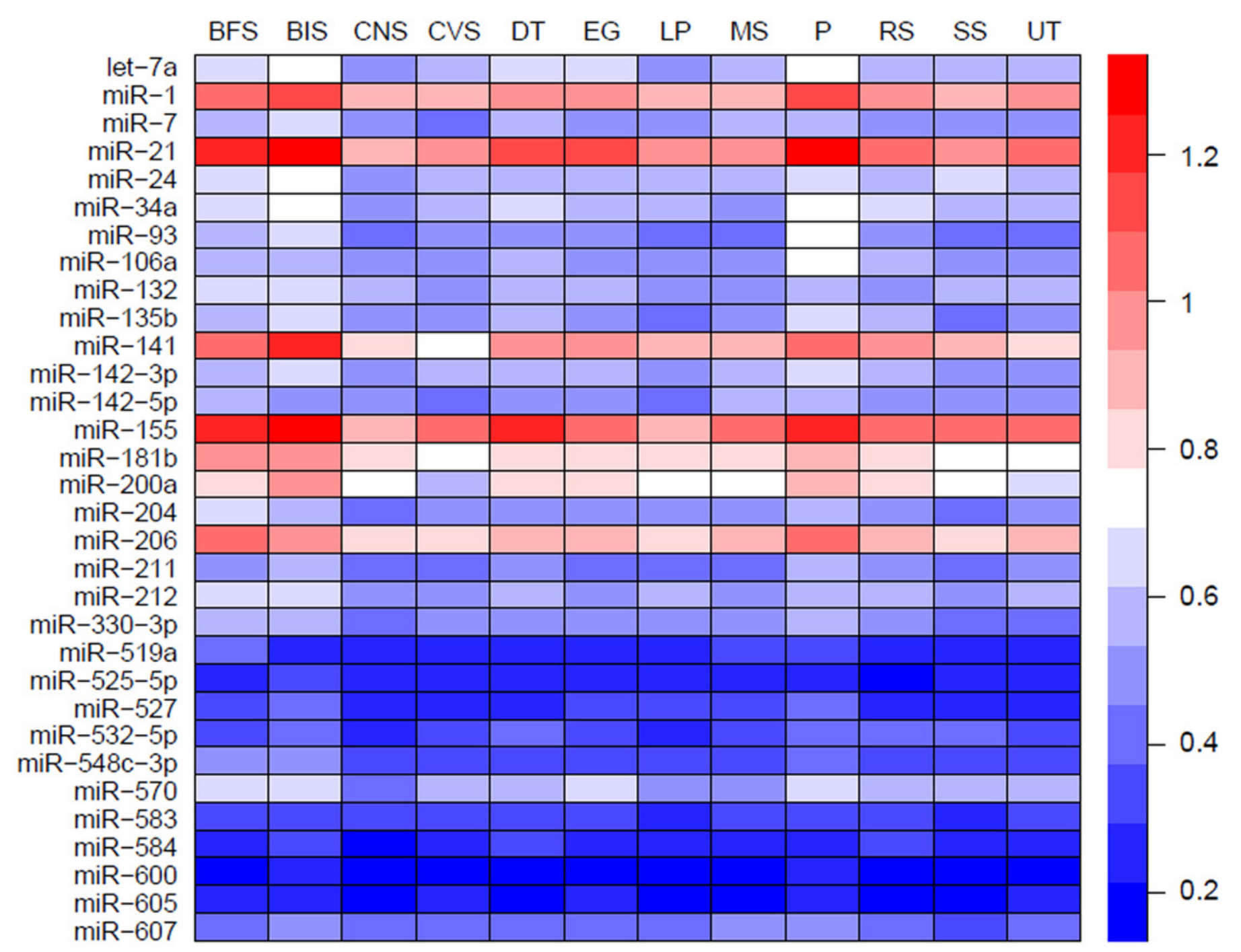


Figure 6

Figure 6. Cell viability assay of miRNA transfection on cell lines of HUVEC (A), MCF7 (B), and HepG2 (C).

** $p<0.01$, versus NC+Hypoxia (HUVEC); $* p<0.05$, versus NC (MCF7 and HepG2); NC: negative control; $\mathrm{n}=6$.

A

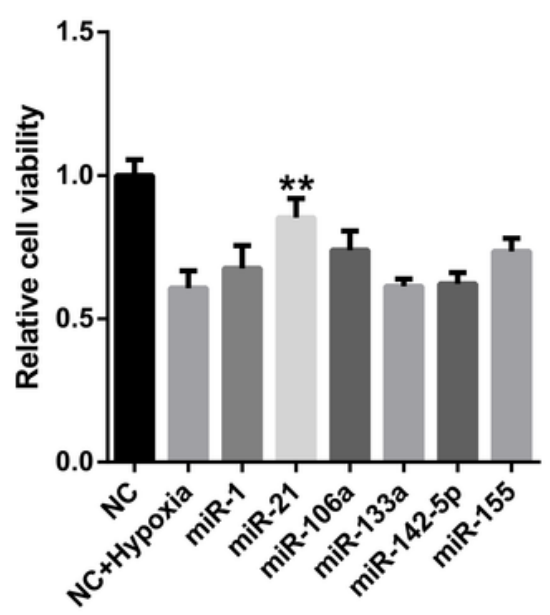

B

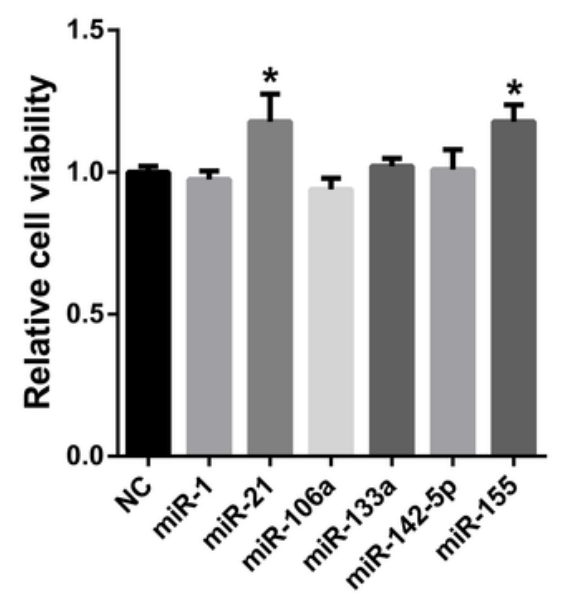

C

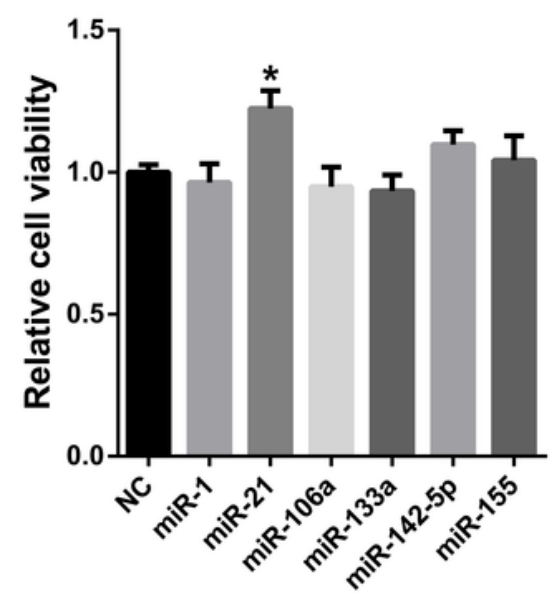

